\title{
Lower Bounds on the Graphical Complexity of Finite-Length LDPC Codes
}

\author{
Igal Sason \\ Department of Electrical Engineering \\ Technion, Haifa 32000, Israel \\ sason@ee.technion.ac.il
}

\begin{abstract}
This paper considers information-theoretic lower bounds on the graphical complexity of finite-length LDPC codes. It is assumed that the transmission of the codes takes place over a memoryless binary-input output-symmetric (MBIOS) channel, and the bounds are expressed as a function of the code performance and their achievable gap to capacity (either under ML decoding or any sub-optimal decoding algorithm). The lower bounds on the graphical complexity are compared to some explicit LDPC codes (or code ensembles), showing that these bounds are informative for considering the fundamental tradeoff which exists between the performance and graphical complexity of finite-length LDPC codes. This work relies on the full paper version [15].
\end{abstract}

Index Terms-Bipartite graphs, complexity, low-density paritycheck (LDPC) codes, memoryless binary-input output-symmetric (MBIOS) channels.

\section{INTRODUCTION}

During the last decade, there have been many exciting developments in the construction of low-complexity errorcorrection codes which closely approach the capacity of many standard communication channels with feasible complexity. These codes are understood to be codes defined on graphs, together with the associated iterative decoding algorithms. By now, there is a large collection of these codes that approach the channel capacity quite closely with moderate complexity.

Khandekar and McEliece suggested to measure the encoding and decoding complexity of codes defined on graphs in terms of the achievable gap (in rate) to capacity, and they also had some conjectures regarding the behavior of the complexity as the gap to capacity vanishes [6]. Following their approach, the tradeoff between the performance and complexity is analyzed in the literature for LDPC code ensembles and some other variants of codes defined on graphs (see, e.g., [4], [5], [8], [9], [12], [14], [20] and references therein).

The fundamental tradeoff between the graphical complexity and performance of codes defined on graphs is of interest, especially for codes of finite-length. In this paper, we address the following question:

Question: Consider the representation of a finite-length binary linear block code by an arbitrary bipartite graph. How simple can such a graphical representation be as a function of the channel model, target block error probability, and code rate (which is below capacity)?

We note that the graphical complexity referred to in this paper measures the total number of edges used for the representation of finite-length codes by bipartite graphs. By referring to the total number of edges, the graphical complexity is strongly related to the decoding complexity per iteration. This differs from the graphical complexity in [2], [4], [8] and [9] which measures the number of edges per information bit in the asymptotic case where we let the block length tend to infinity. Although it may appear at first glance that the aforementioned distinction is just a matter of normalization, this is not the case: The reason is that given the target block error probability and the required gap to capacity for achieving this target with any finite-length block code, one needs first to calculate the minimal block length which potentially allows to fulfill these requirements. It is done in this work via the calculation of classical and recent sphere-packing bounds (see [16], [17], [19] and [21]).

Proofs and further discussions are omitted due to space limitations, and we refer the interested reader to the full paper version [15].

\section{PRELIMINARIES}

\section{A. Sphere-Packing Lower Bounds}

Sphere-packing bounds are commonly used for the study of the performance limitations of finite-length error-correcting codes over memoryless symmetric channels. For a tutorial on classical sphere-packing bounds, the reader is referred to [13, Chapter 5]. This analysis in this paper relies on the following sphere-packing bounds:

- The SP59 bound: The 1959 sphere-packing (SP59) bound of Shannon [16] serves for the evaluation of the performance limits of block codes whose transmission takes place over an AWGN channel. This lower bound on the decoding error probability is expressed in terms of the block length and the rate of the code; however, it does not take into account the modulation used, but only assumes that the modulated signals have equal energy. It is often used as a reference for quantifying the sub-optimality of error-correcting codes under some practical decoding algorithms (see [13, Chapter 5] and references therein). An efficient algorithm for the calculation of the SP59 bound is introduced in [21, Section IV.C].

- The ISP bound: This sphere-packing bound was recently derived in [21, Section III]. The ISP bound applies to all memoryless symmetric channels. For codes of finite block length, it improves the classical sphere-packing bound of 
Shannon, Gallager and Berlekamp [17] and the spherepacking bound of Valembois and Fossorier [19] where this improvement is especially pronounced for short to moderate block lengths. We note that the ISP bound in [21] is not uniformly tighter than the SP59 bound for equi-energy signals transmitted over an AWGN channel.

Comparisons between the sphere-packing bounds in [16], [19] and [21, Section III] are shown in [21, Section V].

\section{B. Tangential-Sphere Upper Bound}

The tangential-sphere bound [10] forms an upper bound on the ML decoding error probability of binary linear block codes which are modulated by equi-energy signals (e.g., PSK signals) and transmitted over an AWGN channel. For a presentation of the tangential-sphere bound, originally introduced by Poltyrev [10], we refer the reader to [13, pp. 23-32].

\section{INFORMATION-THEORETIC BOUNDS}

This section introduces an information-theoretic lower bound which is related to the average right degree of binary linear block codes which are represented by an arbitrary bipartite graph. From the classical sphere-packing bound of Shannon [16], and the recently introduced ISP bound [21], which hold for a general memoryless symmetric channel, one can also obtain a lower bound on the minimal required block length as a function of the target performance. Consider the graphical complexity of binary linear block codes which are represented by an arbitrary bipartite graphs (i.e., the total number of edges in the graph). The graphical complexity scales linearly with product of the block length and the average right degree of the bipartite graph, hence lower bounds on both quantities yield a lower bound on the ultimate tradeoff which exists between the graphical complexity and performance of binary linear block codes which are represented by an arbitrary bipartite graph.

Theorem 1: [On the average degree of the parity-check nodes] Let $\mathcal{C}$ be a binary linear block code of block length $n$ whose transmission takes place over an MBIOS channel. Let $\mathcal{G}$ be a bipartite graph which corresponds to a full-rank paritycheck matrix of $\mathcal{C}$. Let $C$ designate the capacity of the channel, in bits per channel use, and $a$ be the $L$-density function of this channel. Assume that the code rate is (at least) a fraction $1-\varepsilon$ of the channel capacity (where $0<\varepsilon<1$ ), and the code achieves a block error probability $P_{\mathrm{B}}$ or a bit error probability $P_{\mathrm{b}}$ under some decoding algorithm. Then, the average right degree of the bipartite graph (i.e., the average degree of the parity-check nodes in $\mathcal{G}$ ) satisfies

$$
a_{\mathrm{R}} \geq \frac{2 \ln \left(\frac{1}{1-2 h_{2}^{-1}\left(\frac{1-C-\delta}{1-(1-\varepsilon) C}\right)}\right)}{\ln \left(\frac{1}{g_{1}}\right)}
$$

where $g_{1} \triangleq \mathbb{E}\left[\tanh ^{2}(L / 2)\right]$ depends only on the MBIOS communication channel $(L$ is a random variable which refers to the log-likelihood ratio at the channel output, given that the binary input symbol to the channel is zero), and

$\delta \triangleq\left\{\begin{array}{ll}P_{\mathrm{B}}+\frac{h_{2}\left(P_{\mathrm{B}}\right)}{n} & \text { for a block error probability } P_{\mathrm{B}} \\ h_{2}\left(P_{\mathrm{b}}\right) & \text { for a bit error probability } P_{\mathrm{b}}\end{array}\right.$.

Furthermore, among all the MBIOS channels which exhibit a given capacity $C$ and for which a target block error probability $\left(P_{\mathrm{B}}\right)$ or a bit error probability $\left(P_{\mathrm{b}}\right)$ is obtained under some decoding algorithm, a universal lower bound on $a_{\mathrm{R}}$ holds by replacing $g_{1}$ on the RHS of (1) with $C$.

For the BEC, the following tightened version of (1) holds:

$$
a_{\mathrm{R}} \geq \frac{\ln \left(1+\frac{p-P_{\mathrm{b}}}{(1-p) \varepsilon+P_{\mathrm{b}}}\right)}{\ln \left(\frac{1}{1-p}\right)}
$$

where $p$ is the erasure probability of the channel, and $P_{\mathrm{b}}$ is the bit erasure probability at the decoder.

Proof: Due to space limitations, the reader is referred to [15, Section-IV.A] for a proof of this theorem. In general, the proof follows by relying on the Fano inequality, and also on the following lower bound on the conditional entropy of the transmitted codeword given the received sequence at the channel output (see [20]):

$$
\frac{H(\mathbf{X} \mid \mathbf{Y})}{n} \geq R-C+\frac{1-R}{2 \ln 2} \sum_{p=1}^{\infty} \frac{\Gamma\left(g_{p}\right)}{p(2 p-1)}
$$

where

$$
g_{p} \triangleq \int_{0}^{\infty} a(l)\left(1+e^{-l}\right) \tanh ^{2 p}\left(\frac{l}{2}\right) d l, \quad p \in \mathbb{N} .
$$

and where $\Gamma(x) \triangleq \sum_{k} \Gamma_{k} x^{k}$ with $\Gamma_{k}$ designating the fraction of the parity-checks involving $k$ variables in a specific representation of the code by a full-rank parity-check matrix. The bound in (4) holds for any representation of the code by a full-rank parity-check matrix. The validity of (4) is adapted in [15, Appendix I] to every code from an LDPC code ensemble (whose parity-check matrix is not necessarily full rank) by replacing the code rate with the design rate of the ensemble (where the design rate forms a lower bound on the rate of the code). Note that the sequence $\left\{g_{p}\right\}$ in (5) forms a nonincreasing and non-negative sequence (upper bounded by 1) which serves for a fast convergence of the RHS in (4).

Remark 1: [The relation of Theorem 1 to the bound in [20]] In the particular case where $P_{\mathrm{b}}$ vanishes, the bound in (1) forms a tightened version of the bound given in [20, Eq. (77)]. This point is clarified in [15]. In the limit where the gap (in rate) to capacity vanishes (and with vanishing $P_{\mathrm{b}}$ ), the lower bounds on the average right degree in (1) and [20, Eq. (77)] both grow like the logarithm of the inverse of this gap, and they therefore possess the same asymptotic behavior where

$$
a_{\mathrm{R}} \triangleq a_{\mathrm{R}}(\varepsilon)=\Omega\left(\ln \frac{1}{\varepsilon}\right) .
$$

However, in spite of the similarity in the asymptotic behavior of the two lower bounds as $\varepsilon \rightarrow 0$, they may differ significantly even for rather small values of $\varepsilon$ as is shown in the following example: 
Example 1: [Comparison of the lower bound on the average right degree from Theorem 1 with the bound in [20]] In the following, we exemplify the practical use of the lower bound on the average right degree of LDPC code ensembles, as given in Theorem 1 and its adaptation to LDPC code ensembles in [15, Discussion 2], and compare it with the previously reported bound in [20, Section IV]. Consider the case where the communications takes place over a BIAWGNC. The LDPC code ensembles in each sequence are specified by the following pairs of degree distributions, followed by their corresponding design rates and thresholds under BP decoding: Ensemble 1:

$$
\begin{aligned}
& \lambda(x)=x, \quad \rho(x)=x^{19}, \quad R_{\mathrm{d}}=0.9000 . \\
& \sigma_{\mathrm{BP}}=0.4156590 .
\end{aligned}
$$

Ensemble 2:

$$
\begin{aligned}
& \lambda(x)=0.4012 x+0.5981 x^{2}+0.0007 x^{29}, \quad \rho(x)=x^{24} \\
& R_{\mathrm{d}}=0.9000, \quad \sigma_{\mathrm{BP}}=0.4741840 .
\end{aligned}
$$

These code ensembles are taken from the data base in [1]. From [11, Example 4.38] which expresses the capacity of the BIAWGNC in terms of the standard deviation $\sigma$ of the Gaussian noise, the minimum capacity of a BIAWGNC over which it is possible to communicate with vanishing bit error probability under BP decoding is $C=0.9685$ and 0.9323 bits per channel use for Ensembles 1 and 2, respectively. The corresponding gap (in rate) to capacity $\varepsilon=1-\frac{R_{\mathrm{d}}}{C}$ is equal to $\varepsilon=7.07 \cdot 10^{-2}$ and $3.46 \cdot 10^{-2}$, respectively. Therefore, for the first ensemble which is a $(2,20)$ regular LDPC code ensemble, the new lower bound on the average right degree which follows from [15, Discussion 2] is equal to 9.949 whereas the lower bound from [20, Section IV] (i.e., the unnumbered equation before [20, Eq. (77)]) is equal to 2.392. For the second ensemble whose fixed right degree is equal to 25 , the new lower bound on the average right degree is 16.269 whereas the lower bound from [20] is 14.788. This shows that the improvement obtained in Theorem 1 is of practical use.

We note that the gap which still exists between the lower bounds on the average right degrees and the actual values of $a_{\mathrm{R}}$ for the above two ensembles is partially attributed to the fact that this information-theoretic lower bound holds even under ML decoding, although we apply this bound here under the sub-optimal BP decoding algorithm. The gaps to capacity under ML decoding are smaller than those calculated under BP decoding, and smaller values of $\varepsilon$ provide improved lower bounds on $a_{\mathrm{R}}$.

Remark 2: [Adaptation of Theorem 1 to LDPC code ensembles] As is proved in [15, Appendix I], Theorem 1 can be adapted to hold for an arbitrary ensemble of $(n, \lambda, \rho)$ LDPC codes. In this case, the requirement of a full-rank parity-check matrix of a particular code $\mathcal{C}$ from this ensemble is relaxed by requiring that the design rate of the LDPC code ensemble is equal to a fraction $1-\varepsilon$ of the channel capacity. In this case, $P_{\mathrm{b}}$ and $P_{\mathrm{B}}$ stand for the average bit and block error (or erasure) probabilities of the ensemble under some decoding algorithm.

\section{The graphical complexity of finite-length LDPC codes}

In Section IV, we apply Theorem 1 and sphere-packing bounds on the decoding error probability (see [16], [17], [19], [21]) to obtain information-theoretic lower bounds on the graphical complexity of finite-length LDPC codes. These bounds are expressed as a function of the target block error probability and the gap between the design rate of the code and the channel capacity. We note that in this context, the graphical complexity measures the number of edges used for the representation of finite-length codes by bipartite graphs. By referring to the total number of edges, the graphical complexity is strongly related to the decoding complexity per iteration. The bounds are compared with capacity-approaching LDPC code ensembles under BP decoding, and they are shown to be informative (see Section IV).

\section{Numerical Results For the GRAPHicAL COMPLEXITY}

In various applications, there is a need to design a communication system which fulfills several requirements on the available bandwidth, acceptable delay for transmitting and processing the data while maintaining a certain fidelity criterion in reconstructing the data. In this setting, one wishes to design a code which satisfies the delay constraint (i.e., the block length is limited) while adhering to the required performance over the given channel. By fixing the communication channel model, code rate (which is related to the bandwidth expansion caused by the error-correcting code), then sphere-packing bounds are transformed into lower bounds on the minimal block length required to achieve a target block error probability at a certain gap to capacity using an arbitrary block code and decoding algorithm. This issue is studied in [21, Section V].

In the following, we refer to the graphical complexity of an arbitrary bipartite graph which represents a binary linear block code. The graphical complexity has an operational meaning for an iterative message-passing decoder since the number of edges is equal to the number of right-to-left and left-to-right messages which are delivered in each iteration. As opposed to [4], [8] and [9], we refer here to the graphical complexity of finite-length codes. In order to evaluate an informationtheoretic lower bound on the graphical complexity which is expressed in terms of the target block error probability and the corresponding achievable gap to capacity, we rely here on the following algorithm:

- Step 1: Sphere-packing bounds are used to calculate a lower bound on the minimal required block length in terms of the achievable rate with a target block error probability and its gap to capacity. For a memoryless symmetric channel, the lower bound on the minimal block length is calculated via the ISP bound (for finitelength codes, this recent sphere-packing bound suggests a significant improvement over the bounds in [17] and [19], see Section II-A and [21, Section III]). In addition, this lower bound is also compared with the 1959 spherepacking (SP59) bound of Shannon (see Section II-A and [16]) for a binary-input AWGN channel where the transmitted signals are assumed to have equal energy. 


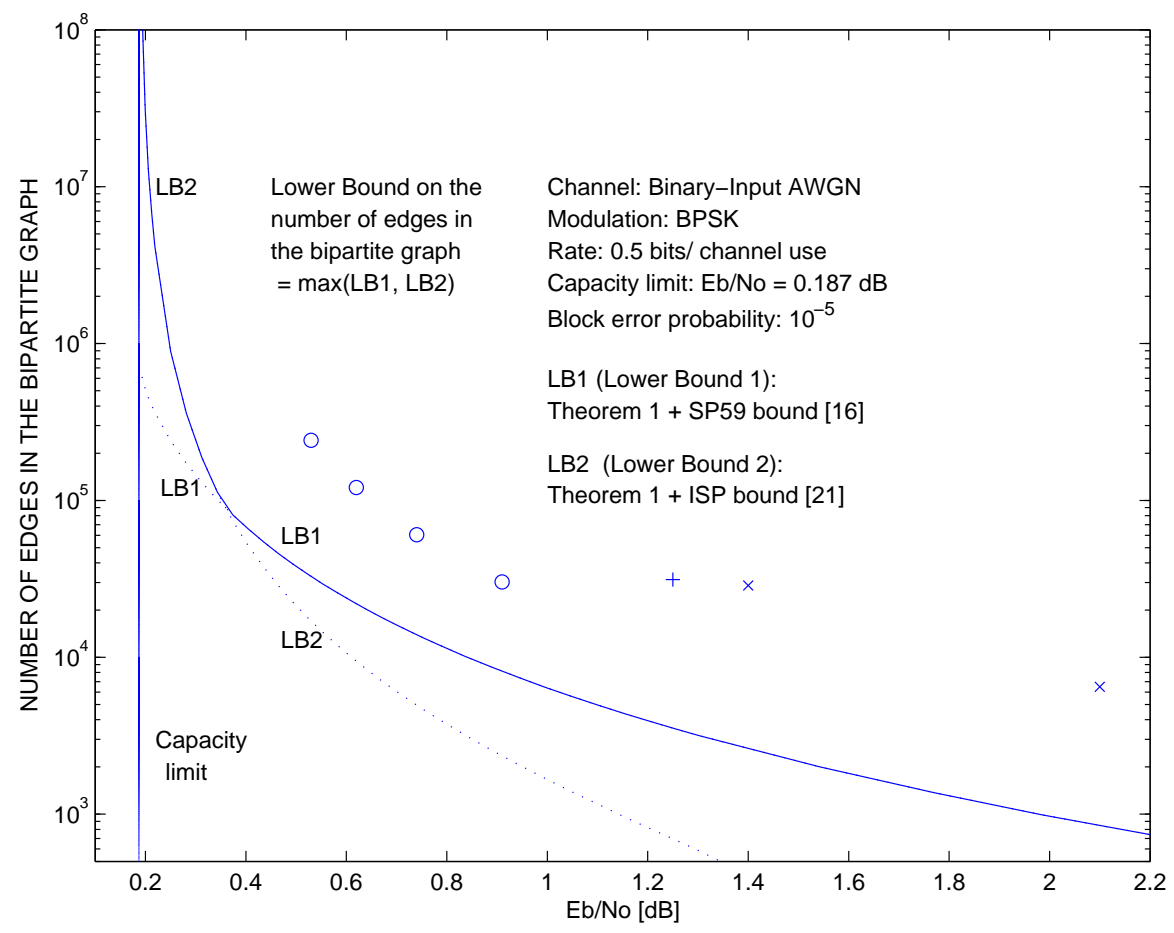

Fig. 1. A comparison between the graphical complexity of various efficient LDPC code ensembles and an information-theoretic lower bound. The graphical complexity is measured by the number of edges which are used to represent the codes (or code ensembles) by bipartite graphs in order to achieve a fixed target block error probability over a given communication channel. It is assumed that the code is BPSK modulated and transmitted over a binary-input AWGN channel. This figure refers to a target block error probability of $P_{\mathrm{B}}=10^{-5}$, and a design rate of one-half bit per channel use. The information-theoretic lower bound is valid under maximum-likelihood (ML) decoding (and, hence, it also holds under any sub-optimal decoding algorithm). For the comparison of the lower bound with various LDPC code ensembles, we refer to both ML and belief-propagation (BP) decoding algorithms. The circled points refer to ML decoding, and they are based on the tangential-sphere upper bound which is applied to the $(6,12)$ regular LDPC code ensembles of Gallager for block lengths of 5040, 10080, 20160 and 40320 bits (these points rely on [18, Table II]). The other three points in this figure refer to LDPC code ensembles which are decoded by a BP decoder. The point marked by ' $+{ }^{\prime}$ refers to a non-punctured protograph LDPC code ensemble of block length 7360 bits and of rate one-half (see [3, Fig. 9]). The other two points which are marked by ' $x$ ' refer to irregular quasi-cyclic LDPC code ensembles (see [7, Figs. 10 and 11]).

- Step 2: A lower bound on the average right degree is calculated via Theorem 1 for an arbitrary bipartite graph which is used to represent a binary linear block code. Note that for an LDPC code whose parity-check matrix is not necessarily full-rank, one can apply this lower bound by replacing the code rate with the design rate (see Discussion 2 in [15, Section IV]). The calculation of this lower bound for a target block error probability $P_{\mathrm{B}}$ also stays valid if the block length $n$ is replaced in (2) with a lower bound $n^{\prime}$ (as calculated in the previous step).

- Step 3: The total number of edges of a bipartite graph is a measure of its graphical complexity. For a bipartite graph which refers to a design rate of $R_{\mathrm{d}}$, the total number of edges is equal to $|\mathcal{E}|=\left(1-R_{\mathrm{d}}\right) n a_{\mathrm{R}}$. Replacing $n$ and $a_{\mathrm{R}}$ by the lower bounds calculated in Steps 1 and 2, respectively, gives a lower bound on the number of edges.

The resulting lower bound on the total number of edges is general for every representation of a binary linear block code by a parity-check matrix and its respective bipartite graph. This bound depends on the code rate (or design rate), the communication channel, the achievable gap to capacity, and the target block error probability. This lower bound holds for an arbitrary representation of the code by a bipartite graph.

According to the above description of the three steps used to calculate the information-theoretic lower bound on the graphical complexity, we calculate here two lower bounds on the graphical complexity:

- LB1: A lower bound which combines a lower bound on the block length calculated via the SP59 bound [16], and a lower bound on the average right degree which is calculated via Theorem 1 for a target block error probability $P_{\mathrm{B}}$ and a given code rate (or design rate).

- LB2: A lower bound which combines a lower bound on the block length calculated via the ISP bound [21, Section III], and the same lower bound on the average right degree.

We note that Steps 2 and 3 in the above algorithm are common for the calculation of the LB1 and LB2 bounds, and the only difference in the calculation of these two bounds is in Step 1 where the SP59 and ISP bounds are used for the LB1 and LB2 bounds, respectively. The resulting lower bound (LB) on the graphical complexity is the maximal value of the LB1 and LB2 bounds, i.e., $\mathrm{LB}=\max (\mathrm{LB} 1, \mathrm{LB} 2)$. We note that the resulting lower bound on the graphical complexity holds under ML decoding or any sub-optimal decoding algorithm.

The above algorithm is applied in Figure 1 to obtain a lower bound on the graphical complexity of an arbitrary binary linear block code of rate one-half and with a target block error 
probability of $P_{\mathrm{B}}=10^{-5}$. It is assumed that the code is BPSK modulated, and the transmission takes place over a binaryinput AWGN channel. The un-bounded complexity in the limit where the gap to capacity vanishes is due to the infinite block length which is required to obtain reliable communications at rates which are arbitrarily close to capacity. We note that the bounded graphical complexity for the BEC, as demonstrated in [4], [8] and [9], is obtained by addressing the graphical complexity per information bit, and by also allowing more complicated Tanner graphs which include state nodes (e.g., punctured bits) in addition to the variable and parity-check nodes which are used for a representation of these codes by bipartite graphs.

As shown in Figure 1, the bound LB2 is advantageous over LB1 for low values of $\frac{E_{\mathrm{b}}}{N_{0}}$ which are close to the capacity limit; this phenomenon is even more pronounced for higher code rates (above one-half bit per channel use). This observation is partially due to the fact that the ISP bound depends on the particular type of modulation used, in contrast to the SP59 bound which only assumes that the modulated signals have equal energy but does not consider the particular modulation used.

The lower bound on the graphical complexity is compared here with some efficient LDPC codes (or code ensembles) as reported in the literature. To this end, we refer to computer simulations under BP decoding, and also to upper bounds on the block error probability under ML decoding. Although the number of edges is relevant for the decoding complexity per iteration under BP decoding, some comparisons with ML decoding provide a better assessment of the tightness of this information-theoretic lower bound. The circled points in Figure 1 are based on the tangential-sphere upper bound which is applied to the $(6,12)$ regular LDPC code ensembles of Gallager for block lengths of 5040, 10080, 20160 and 40320 bits whose block error probability is upper bounded by $10^{-5}$ (see [18, Table II]). The other three points which are shown in Figure 1 refer to LDPC code ensembles which are decoded by a BP decoder. The point marked by ' + ' refers to a non-punctured protograph LDPC code ensemble of block length of 7360 bits and a design rate of one-half (see [3, Fig. 9]). The other two points which are marked by ' $x$ ' refer to irregular quasi-cyclic LDPC code ensembles (see [7, Figs. 10 and 11]) where the graphical complexity is obtained via the degree distributions which are given in [7, Examples 10 and 11]. To conclude, the information-theoretic lower bound on the graphical complexity becomes un-bounded as the gap to capacity vanishes (even under ML decoding). It also behaves in a similar way to the circled points in Figure 1 (where these points refer to the performance of a regular LDPC code ensembles under ML decoding). Moreover, the comparison of this lower bound in Figure 1 with some efficient LDPC code ensembles under BP decoding (where the corresponding points are marked by ' $+{ }^{\prime}$ and ' $x$ ') indicate the gain that can be potentially obtained by improved designs of efficient LDPC codes and iterative decoding algorithms defined on graphs.

\section{REFERENCES}

[1] A. Amraoui and R. Urbanke, LdpcOpt: Software for optimizing the degree distrbutions of LDPC code ensembles. [Online]. Available: http: //lthcwww.epfl.ch/research/ldpcopt/index.php.

[2] M. Ardakani, B. Smith, W. Yu and F. R. Kschischang, "Complexityoptimized low-density parity-check codes," Proceedings of the FortyThird Annual Allerton Conference on Communication, Control and Computing, pp. 45-54, Urbana-Champaign, Illinois, USA, September 2005.

[3] D. Divsalar, S. Dolinar and C. Jones, "Construction of protograph LDPC codes with linear minimum distance," Proceedings 2006 IEEE International Symposium on Information Theory, pp. 664-668, Seattle, Washington, USA, July 6-12, 2006.

[4] C. H. Hsu and A. Anastasopoulos, "Capacity-achieving codes with bounded graphical complexity on noisy channels," Proceedings of the Forty-Third Annual Allerton Conference on Communications, Control and Computing, pp. 1825-1834, Urbana-Champaign, IL, USA, September 2005 .

[5] C. H. Hsu and A. Anastasopoulos, "Capacity-achieving LDPC codes through puncturing," IEEE Trans. on Information Theory, vol. 54, no. 10, pp. 4698-4706, October 2008.

[6] A. Khandekar and R. J. McEliece, "On the complexity of reliable communication on the erasure channel," Proceedings 2001 IEEE International Symposium on Information Theory, p. 1, Washington, D.C., USA, June 2001.

[7] L. Lan, L. Zeng, Y. Tai and L. Chen, "Construction of quasi-cyclic LDPC codes for the AWGN and binary erasure channels: A finite field approach," IEEE Trans. on Information Theory, vol. 53, no. 7, pp. 24292458, July 2007.

[8] H. D. Pfister, I. Sason and R. Urbanke, "Capacity-achieving ensembles for the binary erasure channel with bounded complexity," IEEE Trans. on Information Theory, vol. 51, no. 7, pp. 2352-2379, July 2005.

[9] H. D. Pfister and I. Sason, "Accumulate-repeat-accumulate codes: Capacity-achieving ensembles of systematic codes for the erasure channel with bounded complexity," IEEE Trans. on Information Theory, vol. 53, no. 6, pp. 2088-2115, June 2007.

[10] G. Poltyrev, "Bounds on the decoding error probability of binary linear codes via their spectra," IEEE Trans. on Information Theory, vol. 40, pp. 1284-1292, July 1994.

[11] T. Richardson and R. Urbanke, Modern Coding Theory, Cambridge University Press, 2008. [Online]. Available: http://lthcwww.epfl.ch/mct/ index.php.

[12] I. Sason and R. Urbanke, "Parity-check density versus performance of binary linear block codes over memoryless symmetric channels," IEEE Trans. on Information Theory, vol. 49, no. 7, pp. 1611-1635, July 2003.

[13] I. Sason and S. Shamai, "Performance Analysis of Linear Codes under Maximum-Likelihood Decoding: A Tutorial," Foundations and Trends in Communications and Information Theory, vol. 3, no. 1-2, pp. 1-222, Now Publishers, Delft, the Netherlands, July 2006. [Online]. Available: http://www.ee.technion.ac.il/people/sason/monograph_postprint.pdf.

[14] I. Sason and G. Wiechman, "Bounds on the number of iterations for turbo-like ensembles over the binary erasure channel," IEEE Trans. on Information Theory, vol. 55, no. 6, June 2009.

[15] I. Sason, "On universal properties of capacity-approaching LDPC code ensembles," IEEE Trans. on Information Theory, vol. 55, no. 7, July 2009.

[16] C. E. Shannon, "Probability of error for optimal codes in a Gaussian channel," Bell System Technical Journal, vol. 38, pp. 611-656, May 1959.

[17] C. Shannon, R. Gallager and E. Berlekamp, "Lower bounds to error probability for decoding on discrete memoryless channels," Information and Control, vol. 10, Part 1: pp. 65-103, and Part 2: pp. 522-552, February/May 1967.

[18] S. Tong, "Tangential-sphere bounds on the ensemble performance of ML decoded Gallager codes via their exact ensemble distance spectrum," Proceedings 2008 IEEE International Symposium on Communications, pp. 1150-1154, Beijing, China, May 2008.

[19] A. Valembois and M. Fossorier, "Sphere-packing bounds revisited for moderate block length," IEEE Trans. on Information Theory, vol. 50, no. 12, pp. 2998-3014, December 2004.

[20] G. Wiechman and I. Sason, "Parity-check density versus performance of binary linear block codes: New bounds and applications," IEEE Trans. on Information Theory, vol. 53, no. 2, pp. 550-579, February 2007.

[21] G. Wiechman and I. Sason, "An improved sphere-packing bound on the performance of finite-length error-correcting codes," IEEE Trans. on Information Theory, vol. 54, no. 5, pp. 1962-1990, May 2008. 